\title{
Regulatory crosstalk of the metabolic network
}

\author{
Nana-Maria Grüning, Hans Lehrach and Markus Ralser
}

Max Planck Institute for Molecular Genetics, Ihnestrasse 73, 14195 Berlin, Germany

The metabolic network has a modular architecture, is robust to perturbations, and responds to biological stimuli and environmental conditions. Through monitoring by metabolite responsive macromolecules, metabolic pathways interact with the transcriptome and proteome. Whereas pathway interconnecting cofactors and substrates report on the overall state of the network, specialised intermediates measure the activity of individual functional units. Transitions in the network affect many of these regulatory metabolites, facilitating the parallel regulation of the timing and control of diverse biological processes. The metabolic network controls its own balance, chromatin structure and the biosynthesis of molecular cofactors; moreover, metabolic shifts are crucial in the response to oxidative stress and play a regulatory role in cancer.

Evolution of the metabolic network and its structure Life probably began with the formation of compartmentalised autocatalytic chemical cycles. With the appearance of complex catalysts (ribonucleic acid- or protein-based enzymes), these cycles gained complexity, and evolved in their effectiveness and robustness [1]. These metabolic pathways now form the basis for life.

As was the case for the first primitive life forms, the survival of today's complex organisms depends on the robustness and functionality of the metabolic framework [2]. To prevent and counteract perturbations in the flux, many biological control and sensor systems have evolved around the metabolic network. Metabolic pathways provide the cell with energy and supply macromolecular synthesis pathways with their required intermediates. They also balance metabolic systems under a variety of physiological conditions, and act as transducers and sensor systems for environmental conditions and stimuli. In addition, metabolic pathways are essential for crosstalk between metabolic regulatory components and the cellular transcriptome and proteome.

As early as the 1960s, the principle that cells alter their gene expression in response to metabolic requirements has been known. The seminal work of Jacob and Monod, investigating the lac operon, uncovered one of the first examples of chemical-genetic regulation, by which bacterial cells adjust their enzyme production to the nutrient supply [3] . However, only recently have the broader implications of this principle emerged. Indeed, changes in the metabolic network not only control the metabolome, but they also have wide-ranging regulatory functions throughout biology.

Corresponding author: Ralser, M. (ralser@molgen.mpg.de)
Most of the biochemical mechanisms that link the metabolic network to the transcriptome and proteome are incompletely understood. However, one type of regulation appears to be common: representative network intermediates bind to and modulate sensor function and activity of transcription factors, translational regulators, chromatin, enzymes, RNA molecules, and ion channels. Significant transcriptional changes around these intermediates identified them as reporter metabolites [4,5]. The use of intermediates as activity reporters necessitates a modular structure of the metabolic network; a semi-independent flux of its substructures is an underlying premise for this type of regulation. Reporter metabolites bridge the gap between the small molecular and macromolecular universe of the cell. Understanding their regulatory crosstalk is of vital interest as they are key components of metabolic pathways affected in human aging and disease.

\section{The metabolic network is modular and robust}

The metabolic network can be subdivided into several small, highly connected functional units, termed metabolic modules [6,7]. Delineation of modular structures is biased by scope and experimental conditions, but genome-scale network reconstructions facilitate the integration of multiple hierarchical layers; they refine module borders $[8,9]$. Network size markedly determines modular organisation; the larger the network, the greater the modularity [10]. Moreover, modularity is a driving force for self-organisation of networks and a basis for functional adaptation [6,10-12].

Adaptation to multiple environmental conditions often requires functional redundancy. For instance, methanotrophic bacteria use different modules to distribute toxic formaldehyde when they switch into or out of methylotrophy [13]. Indeed, environmental transitions could be the driving force for the evolution of modularity. Mathematical simulations revealed that periodic changes in metabolic

\footnotetext{
Glossary

Biochemical pathway: sequence of enzymatic reactions that convert a starting molecule to an end product

Metabolic flux: amount/rate of molecules converted through a metabolic pathway

Metabolic network: Set and topology of metabolic biochemical reactions within a cell

Metabolic switch: enzyme at the boundary between metabolic modules; a change in the activity of the switch redistributes the metabolic flux

Metabolic transition: change in a module's activity, or a switch of the flux from one module to another

Module: functional and/or regulatory unit within the metabolic network

Reporter metabolite: metabolite whose concentration is monitored by sensing

structures (e.g. chromatin), and surrounded by transcriptional changes
} 
requirements drive the evolution of modular structures [14]. Moreover, bacteria that live in complex environments, such as soil, possess greater network modularity than do bacterial parasites that live in constant environments [15].

Modularity facilitates the stability and robustness of the metabolic network because perturbations can be balanced within individual modules without disrupting the flux of the entire system. Experimentally, this idea is supported by large scale surveys of the Saccharomyces cerevisiae and Escherichia coli metabolomes [16,17]. In yeast, $75 \%$ of enzyme disruptions have no detectable effect on the overall metabolic flux [18]. Although this figure is biased, because of the large number of paralogous enzymes [18] and varied experimental conditions [19] it nonetheless indicates the remarkable robustness of the metabolic network. The deletion of most paralogue-free enzyme-encoding genes indeed causes changes in the metabolic flux $[16,18]$, but at least in bacteria and yeast, most perturbations are only detected within a surprisingly narrow part of the network $[17,18]$. A low number of enzyme disruptions, however, cause a system-wide response. In general, these perturbations affect the concentration of common, network interconnecting cofactors or substrates $[16,18]$.

\section{Metabolic modules are connected to regulatory processes}

Under steady state conditions of an idealised metabolic network, different interconnected modules operate at varying concentrations and kinetics (Figure 1). When the conditions change, a reconfiguration of the network might be required. For instance, when cells are environmentally deprived of an amino acid, such as leucine, the endogenous synthesis pathway must be activated. The relevant module then undergoes a transition to increase activity. Such metabolic transitions require the cooperation of multiple regulatory components. For example, following leucine deprivation, its concentration and turnover must be monitored and the synthesis pathway activated. This is achieved by post-translational stimulation of enzymatic activity (common in central metabolism [20]), by transcriptional regulation, or by stabilisation of mRNA or protein levels. Notably, the supply of network-interconnecting metabolites [e.g., ATP or $\mathrm{NAD}(\mathrm{H})$ ] must be rapidly adjusted, as alterations in their concentration would disturb the entire network.

Hence, interactions of the metabolic network with cellular regulatory components are very common. This property is observable through comparative metabolome, transcriptome and proteome analyses. Bacteria, for example, counteract perturbations via changes in their overall mRNA levels, protein content and growth rate; however, they maintain the concentration of central metabolites. Perturbations cause very specific and precise adaptations of the cellular enzyme content, indicating that monitoring of the network occurs at multiple nodes [17]. Balancing the perturbation is then manifold; consequences range from fine-tuning of a few enzymes up to adjusting the overall growth rate $[17,20-23]$. The latter is energetically expensive; a change in the growth rate requires numerous metabolic transitions, and the entire transcriptome, proteome and metabolome must be adapted [23].

\section{Reporter metabolites crosstalk with the cellular regulome}

Patil and Nielsen identified reporter metabolites based on an enrichment analysis of transcriptional changes; they are nodes balanced by the transcriptome $[4,5]$. This definition can be extended, as more recent studies show that



Figure 1. The modular structure of the metabolic network. Schematic illustration of an idealised metabolic network. (i) Functional modules (blue, yellow and green) have different activities and are strongly interconnected by a low number of general cofactors/enzymatic substrates. (ii) A change in the environment or a biological stimulus can prompt a specific module to operate at higher activity. This metabolic transition causes reconfiguration of the network. (iii) The concentration of general metabolites must be balanced, the flux of the network stabilised, and the enzyme content and activity of the target module adjusted. 


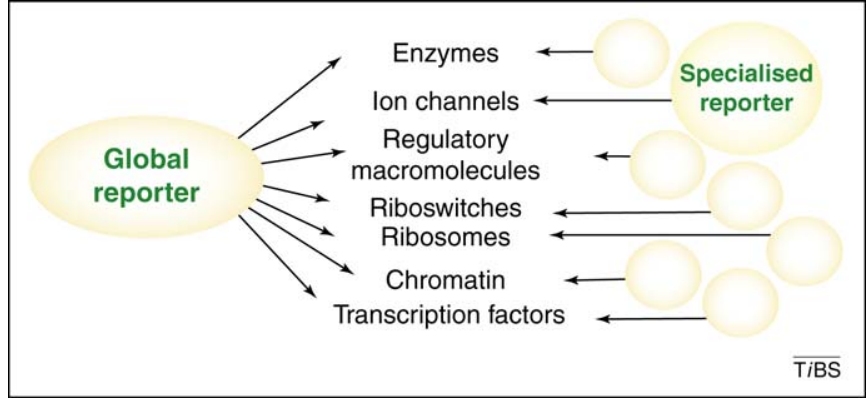

Figure 2. Regulatory metabolites are found among two categories of network intermediates. Global (i.e. general and network interconnecting) and specialized (i.e. pathway specific) reporters connect the metabolic network to cellular regulatory processes. These intermediates are monitored and bind transcription factors, riboswitches and other regulatory macromolecules. These interactions are used to fine-tune the activity of metabolic modules, to detect network perturbations and to facilitate the functioning of metabolic pathways in signalling cascades.

the metabolic flux is often controlled purely on a posttranslational basis [20,24]. The balance of metabolite concentrations requires their constant monitoring by cellular regulatory components. However, differences can be observed, depending on whether the monitored molecule is a network interconnecting agent or a module-specific agent. Both categories function as essential, but distinct, messengers of biological signals (Figure 2).

\section{Network interconnecting metabolites report the overall state of metabolism}

Some cofactors or enzymatic substrates bind to many different enzymes in multiple modules. These molecules [e.g. ATP, NAD(H), S-adenosylmethionine (SAM)] are responsible for most of the interconnectivity within the metabolic network [4]. A change in these metabolites does not allow tracking a perturbation. However, they reflect the overall metabolic state, and indicate broad perturbation and alterations in the environment, or a crucial nutrient status.

A decrease in the cellular ATP level or ATP:AMP ratio indicates a crucial shortage of energy. This serious alarm signal is immediately recognised by complex regulatory systems such as the target of rapamycin (TOR) kinase and AMP-activated protein kinase signalling pathways [25,26]. Their cooperative activity blocks the cell cycle, prevents ribosome biogenesis, activates compensatory systems (such as autophagy), and increases nutrient uptake and mitochondrial activity. In sum, they halt cellular growth and prevent a collapse of the energy state (see recent reviews $[25,26])$.

Changes in the growth rate are crucial for a cell; they entail a fundamental rearrangement of the cellular physiology and are energetically very costly [23]. It is therefore advantageous to be able to predict, in advance, the requirements for such changes. Mitochondrial back signalling was recently described as a cascade that monitors respiratory energy production [27]. A decrease in mitochondrial activity, which is indicative of a drop in cellular energy, is used as a signal to inhibit ribosomal biogenesis. Because ribosomal biogenesis and protein translation are the most energy-consuming processes of the cell [28], their shutdown anticipates energetic bottlenecks. A yeast strain lacking the mitochondrial ribosomal component Afo1p is deficient for mitochondrial back signalling and respiration, and - as long as fermentable carbon sources are available grows normally. Interestingly, this strain is massively long-living and displays an increase of $60 \%$ in median replicative lifespan [27].

How is the overall state of the network monitored? The aforementioned responses are stress reactions that are activated only when the network reaches a critical stage. However, the regulome often is confronted with more subtle changes, which occur during the cellular aging process or upon slight changes in nutrient supply. In these instances, chromatin takes centre stage in spreading the metabolic signals.

The structure of chromatin is highly dynamic and responsive to post-translational modifications of its integral components (Figure 3). Many chromatin modifications

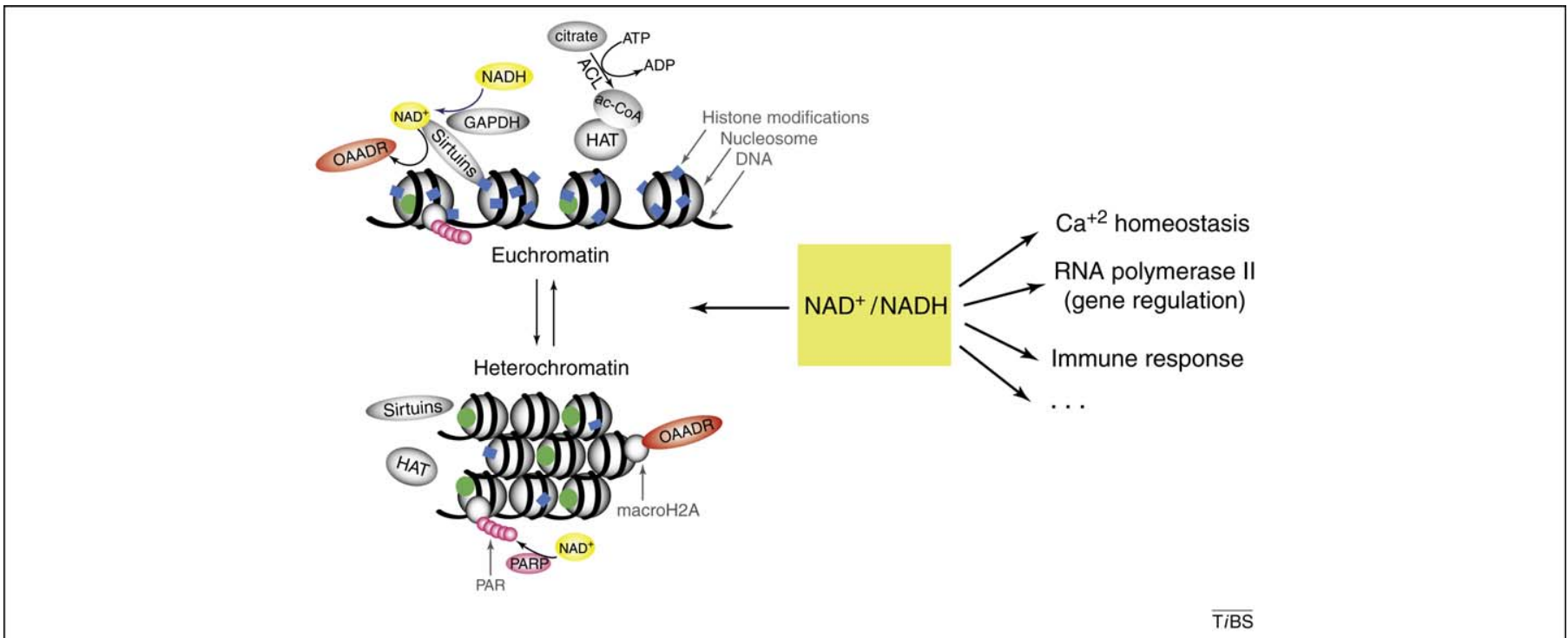

Figure 3. Global metabolic regulators target chromatin. NAD(H) exemplifies a network interconnecting molecule which, as an indicator of broad physiological changes, is involved in multiple regulatory processes. Histone deacetylation, and poly-ADP-ribosylation-dependent methylation, depend on $\mathrm{NAD}^{+}$:NADH; by contrast, histone acetylation is a direct function of metabolic activity. Furthermore, regulatory processes of NAD(H) have been implicated in calcium signalling, transcriptional regulation and the immune response. OAADR, O-acetyl-ADP-ribose. 
are triggered by classic components of the metabolic network. A recent report showed that histone acetyltransferases (HATs) fall under metabolic control, as they are dynamically regulated by physiologic changes in acetylcoenzyme (Co)A concentration. In metazoans, acetyl-CoA, which is derived from glucose and produced by ATP citrate lyase (ACL), is the only HAT acetyl donor. Thus, ACL links nutrient uptake and metabolism to the regulation of histone acetylation, thereby stimulating the expression of glucose metabolism factors [29].

Histone deacetylases (HDACs) also are closely linked to the metabolic network. The fungal secondary metabolome, for instance, changes dramatically upon treatment with HDAC inhibitors [30]. The activity of class III HDACs, the sirtuins, is limited by the availability of $\mathrm{NAD}^{+}$, whose $\mathrm{ADP}$ ribose moiety is used as acetyl group acceptor [31,32]. The yeast sirtuin, Sir2p, forms a complex and interacts genetically with the glycolytic enzyme glyceraldehyde-3-phosphate dehydrogenase (GAPDH) [33,34], an NAD(H)dependent oxidoreductase. In this close proximity to Sir2p, GAPDH can provide or limit NAD depending on the cellular metabolic flux [35]. Sir2p is also stimulated by the $\mathrm{NAD}^{+}: \mathrm{NADH}$ ratio when cells are grown under conditions of mild calorie restriction, and both calorie restriction and increased Sir2 $p$ deacetylase activity can extend lifespan $[32,36]$.

In addition of being targeted by metabolic activity, $\mathrm{NAD}(\mathrm{H})$ concentration and redox state is controlled by the activity of the NAD salvage pathway, the activity of NAD kinases and the synthesis of precursor molecules $[37,38]$. Furthermore, the $\mathrm{NAD}^{+}: \mathrm{NADH}$ ratio responds directly to stress situations [37]. Poly(ADP)ribosylation (PAR), for instance, is a stress-activated, post-translational modification of chromatin-associated proteins. PAR is produced by the enzyme poly(ADP-ribose) polymerase 1 (PARP1), which is highly active and depletes nuclear $\mathrm{NAD}^{+}$during poly(ADP)-ribose synthesis [39]. These events have regulatory consequences in cases of DNA damage. PARP1 activation recruits histone macroH2A and the chromatin remodelling enzyme ALC1 to the site of damage. Around the damaged site they facilitate sitespecific DNA repair via chromatin relaxation $[40,41]$.

$\mathrm{NAD}(\mathrm{H})$ exemplifies the system-wide regulatory consequences that follow changes in the concentration of an interconnecting metabolite (Figure 3). The $\mathrm{NAD}^{+}: \mathrm{NADH}$ ratio influences chromatin-independent signalling events and modulates calcium homeostasis, gene expression (for instance, by targeting RNA polymerase II through PARP) and the immune response (via nuclear factor $\kappa \mathrm{B}$ signalling) $[37,38]$.

\section{Module-specific reporter metabolites adjust transcription cooperatively}

In sharp contrast to the overall regulators, the existence of module-specific reporter metabolites facilitates monitoring of distinct parts of the network. Known sensing mechanisms involve one or more metabolite-responsive protein or RNA molecules, which bind intermediates of the monitored pathway. Metabolite-binding RNA molecules, such as riboswitches, are common and essential in prokaryotes. Riboswitches are RNA motifs located in the $5^{\prime}$ region of mRNAs. Binding of a metabolite induces structural changes and modifies the expression of the downstream coding sequence $[42,43]$. The thiamine-pyrophosphate (TPP) riboswitch, for instance, is found within all kingdoms [44]. TPP, (a phosphorylated derivate of vitamin B1) a widespread coenzyme of all living systems, binds so-called THI boxes. In this way, it fine-tunes translation of factors involved in its own biosynthesis [44]. However, riboswitches seem to play a minor role in eukaryotic cells, especially in metazoans. Instead, in these organisms, the sensing of metabolites via protein-small molecule interactions predominates.

Metabolic intermediates can act as transcriptional feedforward activators. When calorie-restricted yeast is supplemented with fresh nutrients, growth accelerates quickly; as a result, ATP turnover increases rapidly [45]. However, a decrease in ATP cannot be tolerated, because an activated cellular stress program would hinder further growth $[25,26]$. Yeast solves this problem by fast and

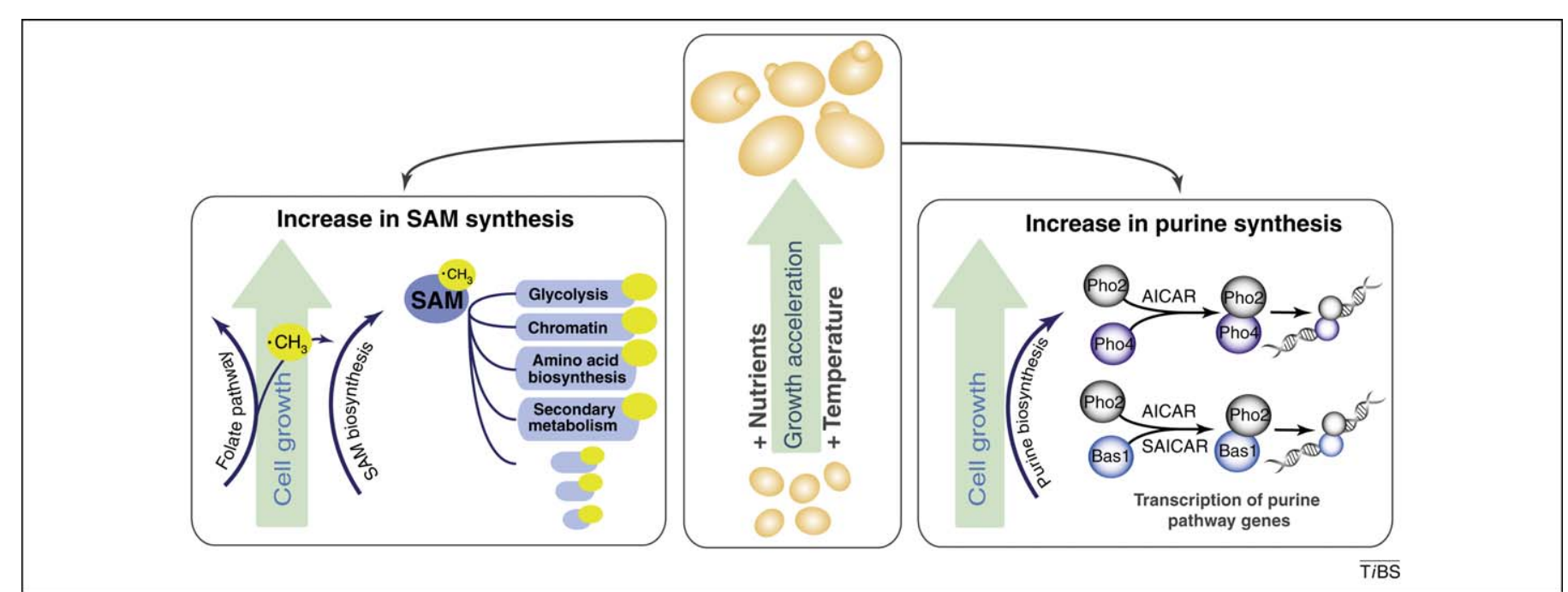

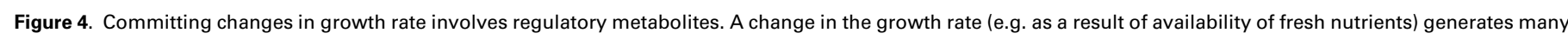

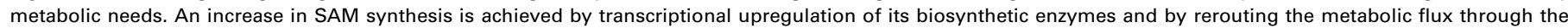


Pho2p-Pho4p) that activate expression of purine synthesis and phosphate utilisation pathways. 
anticipatory upregulation of purine biosynthesis and salvage enzymes [46]. Two intermediates, AICAR (5'-phosphoribosyl-5-amino-4-imidazole carboxamide) and SAICAR (succinyl-AICAR), initiate this regulatory adaptation (Figure 4). AICAR and SAICAR bind the transcription factors Pho2p and Pho4p. Whereas binding of AICAR stimulates the formation of a Pho2p-Pho4p heterodimer, binding of AICAR and SAICAR directs Pho2p-Bas1p binding. These newly formed transcription factor complexes cooperatively induce the expression of genes involved in purine biosynthesis and phosphate utilization pathways [47]. The rationale for a differential response to changes in AICAR and SAICAR could be based on the dual function of these metabolites in inosine monophosphate (IMP) and AMP biosynthesis; the assembly of two competing transcription factor complexes could facilitate a differential response to changes in the de novo pathway and altered GMP:AMP ratios [47,48].

Some molecules can function as both general and specific regulators. Here, SAM provides an illustrative example. SAM, a methyl group donor and one of the most interconnected metabolites of the cell, is a general reporter of nutrient supply. At the same time it is directly involved in sensing and stimulating its own biosynthesis. SAM turnover is highly dependent on the cellular growth rate. In yeast, growth stimuli cooperatively upregulate SAM biosynthetic enzymes. Furthermore, it redirects the metabolic flux through the folate synthesis pathway and increases the production of the SAM precursor, 5methyl-tetrahydropteroyltriglutamate [23]. SAM plays an interesting dual function in the pathogenic bacterium Listeria monocytogenes, where its synthesis is monitored by up to seven classic riboswitches, which sense the SAM concentration and modulate the expression of methionine/ cysteine transporters and metabolic enzymes [49,50]. At least two of the SAM riboswitches play a second role and function as non-coding (nc)RNAs that stimulate the expression of the virulence regulator PrfA and downstream genes [50]. Thus, these SAM- binding RNA molecules perform dual functions: as riboswitches, they specifically balance SAM biosynthesis, whereas as ncRNAs, they regulate the bacterial life cycle.

\section{The importance of self-regulation and transitions in central carbohydrate metabolism}

Pathways of central carbohydrate metabolism, glycolysis in particular, are well-studied examples of highly flexible metabolic systems. The intermediates and side reactions of these pathways are involved in a variety of regulatory processes (Figure 5). Feedback regulation has been extensively studied in Bacillus subtilis, which monitors its dextrose supply through fructose-1,6-bisphosphate (F16BP). F16BP levels increase rapidly upon glucose uptake, thereby antagonising a transcriptional repressor, the central glycolytic genes regulator (CggR). This, in turn, activates an operon that encodes five glycolytic enzymes (gapA, $p g k$,

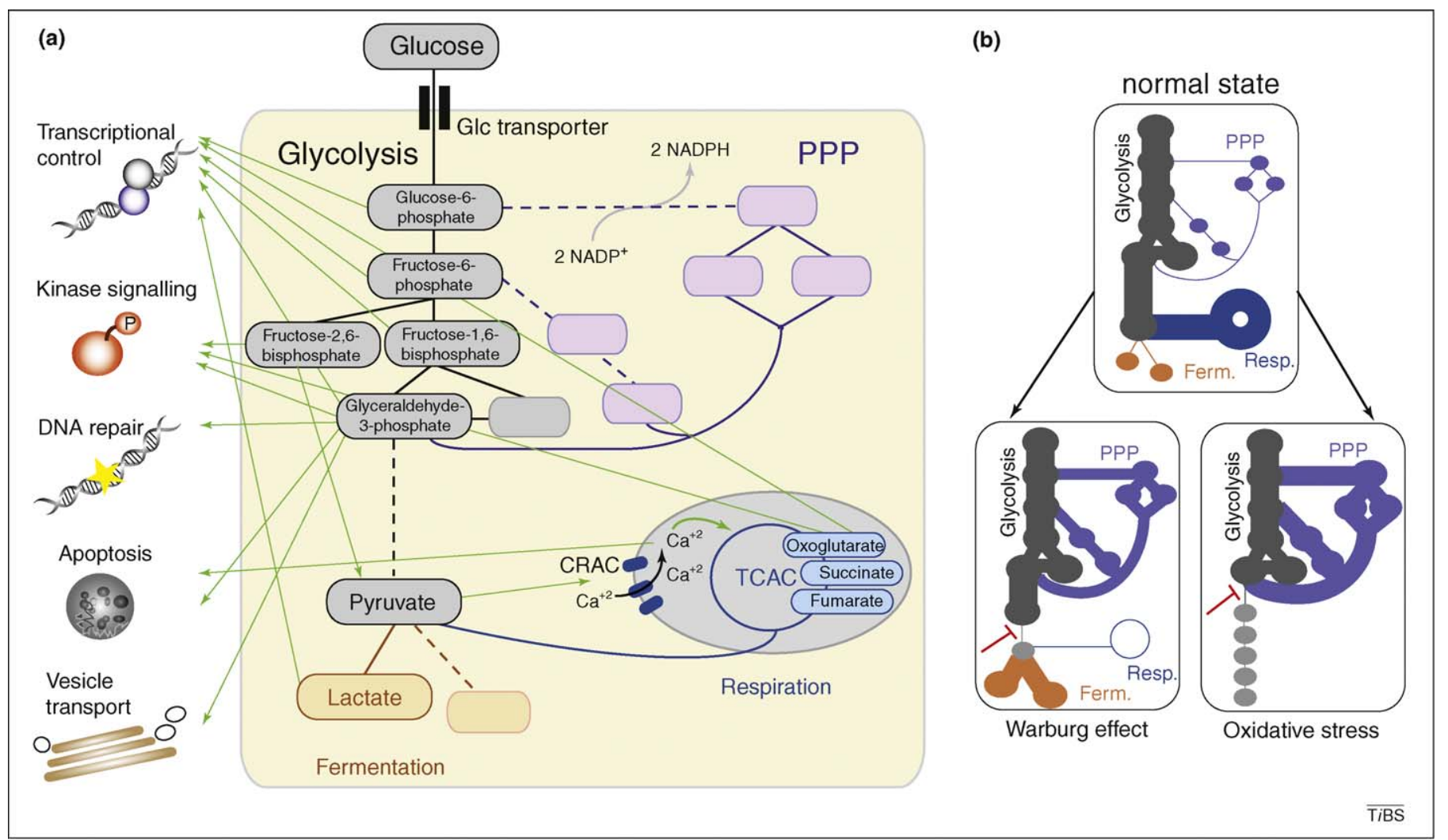

Figure 5. Transitions in central carbohydrate metabolism regulate multiple biological pathways and accompany tumour formation and oxidative stress. Multiple intermediates of central metabolism have reporter functions and spread regulatory signals upon a metabolic shift. (a) Scheme of glycolysis, the pentose phosphate pathway (PPP), tricarboxylic acid cycle (TCA cycle) and respiration. Labelled are metabolites with known regulatory function. Green arrows connect the metabolites to the biological process they regulate. (b) Two transitions affect multiple reporter metabolites within glycolysis. Glycolysis is depicted in grey, reaction of lactate metabolism in orange, the PPP in purple and respiration in blue. Cancer cells with inhibited respiration (Warburg effect) express an alternative pyruvate kinase splice form with reduced activity (left). When exposed to oxidants, cells deviate the carbohydrate flux from glycolysis to the PPP to increase the rate of NADPH recycling (right). 
tpi, pgm and eno). Although F16BP is the main effector, other intermediates (e.g. dihydroxyacetone-phosphate) can also release CggR. Enzymes involved in gluconeogenesis remain repressed as long as glycolytic carbon sources are available; in the absence of such carbon sources, the transcriptional repressor catabolite control protein $\mathrm{N}$ is released, and expression of the gluconeogenetic enzymes is activated [51,52].

\section{The glycolytic flux regulates multiple biological processes}

What is the advantage of monitoring multiple reporters within a single module? First, doing so allows tight control and precise fine-tuning of the transcriptome and proteome. Second, the existence of multiple reporters facilitates the function of metabolic pathways as sensors for biological stimuli and for the timing of biological processes.

The regulatory capacities of the glycolytic flux are not restricted to its self-regulation. In this pathway, reporter metabolite function is the rule rather than the exception (Figure 5); a change in the flux or a metabolic transition influences multiple biological processes by changing the concentration of these metabolites. Under conditions that cause metabolic pathway oscillations, the magnitude of cellular consequences is evident: in yeast, over half of the transcriptome is expressed as a direct function of a metabolic cycle and genes with common functions have similar temporal expression patterns [53].

\section{Reporter metabolites and the Warburg effect}

A specific transition in central carbohydrate metabolism accompanies oncogenic transformations. Otto Warburg noticed as early as the first half of the 20th century that malignant tissues cease respiration, but instead excrete lactate. Today, it has become clear that cancer cells indeed consume higher amounts of glucose and acidify their environment with lactate. It should be noted, however, that cancer cells possess a functional respiratory chain and use mitochondrial metabolism for energy production, albeit at low efficiency $[54,55]$. Neither the reasons for the existence of the Warburg effect, nor its regulatory mechanisms are sufficiently understood. Recent results, however, indicate that the underlying metabolic transition (the Warburg transition) could be essential for cancer cells to survive outside their natural extracellular matrix (ECM) niches. Rerouting of the carbohydrate flux from glycolysis to the pentose phosphate pathway (PPP) is essential for survival of detached cells and is independent of their energy status [56].

The Warburg transition appears to be controlled by a glycolytic enzyme, pyruvate kinase (PK), which acts as a metabolic switch. Transformed cells express an alternative splice form of pyruvate kinase (PKM2), which is usually restricted to embryonic and proliferating tissue and displays reduced catalytic activity [57]. In mouse xenograft experiments, altering the PK expression pattern so that cancer cells expressed only the PKM1 isoform dramatically slowed tumour progression and reactivated respiration [58].

It might seem paradoxical that a reduction in $\mathrm{PK}$ activity should increase lactate excretion, as the conversion of phosphoenolpyruvate (PEP) to pyruvate precedes the formation of lactate. However, reduced PK activity changes the metabolic flux. Loss of bacterial PK, for instance, causes major metabolic rearrangements in glycolysis and elevates flux through the hexose monophosphate pathway and phosphoenolpyruvate carboxylase. Moreover, the PEP:pyruvate ratio increases and dihydroxyacetone and glycerol levels rise [59,60]. Several of the affected metabolites are known regulators of mitochondrial and energy metabolism, and cancer-related signalling systems (Figure 5). Pyruvate, for instance, activates calcium release-activated calcium channels. $\mathrm{Ca}^{2+}$ influx into mitochondria stimulates respiratory metabolism [61] and is crucial for the function of the apoptotic machinery [62]. In addition, lactate also functions as a reporter. It controls the expression of mitochondrial factors and activates master genes of metabolic regulation, including nuclear respiratory factor- 2 and the peroxisome proliferator activated-receptor $\gamma$ coactivator-1 $\alpha$ [63].

Other targeted metabolites that have been implicated in cancer-related signalling pathways include fumarate, succinate, oxoglutarate, fructose-2,6-bisphosphate (F26BP) and glyceraldehyde-3-phosphate. Fumarate, succinate and oxoglutarate stimulate the activity of hypoxia inducible factor 1, a major transcriptional regulator of anaerobic and cancer metabolism [55]. F26BP, which is produced in a glycolytic side reaction, is stimulated by several oncogenic alterations, most likely as a way to over-ride the ATPmediated inhibition of phosphofructokinase. It has further been suggested that selective depletion of F26BP in cancer cells decreases their survival, growth and invasiveness [64].

Recently, glyceraldehyde-3-phosphate joined the 'club' of glycolytic intermediates that are known to stimulate cancer-related signalling cascades. Binding of this metabolite to the glycolytic enzyme GAPDH relieves a block in the mammalian (m)TOR cascade by releasing the RHEB, an mTOR activator [65]. Under high growth rates, this system could be central to prevent mTOR activation and subsequent cell cycle arrest.

Thus, a block of glycolysis at the step of PK, accompanying the Warburg transition, targets multiple reporter metabolites of central carbohydrate metabolism. Many of these have an established function in controlling and modulating the activity of cancer-related signalling pathways, and in stimulating energy metabolism and apoptosis.

\section{Metabolic transitions mediate cellular adaptation}

Balancing the redox state (the ratio between oxidised and reduced molecules) is crucial for biological systems, because the redox potential is a driving force for biochemical reactions and because oxidative imbalances can damage macromolecules. A transition in central carbohydrate metabolism functions as an immediate stabiliser of the redox state. Neutralisation of free radicals largely depends on NADPH, which is required for the function of important effectors of the antioxidant machinery. These include the glutathione, glutaredoxin and thioredoxin systems [38]. To supply enough NADPH during oxidant exposure, cells redirect the carbohydrate flux from glycolysis to the PPP. This change yields two molecules of NADPH 
for every glucose equivalent, but is energetically costly $[66,67]$. The redirection is mediated by two different mechanisms that, interestingly, follow each other over time. Initially, GAPDH acts as a metabolic switch upon inactivation and reroutes the metabolic flux by blocking glycolysis, whereas glucose transporters and the PPP remain active $[68,69]$. This process is very fast; GAPDH is inactivated within seconds and PPP metabolites increase almost immediately [24]. A few minutes later, a genetic response is induced, by which the PPP is upregulated and glycolytic enzymes are downregulated [70]. After the transcriptional reconfiguration of the pathway is complete $(\sim 30-60 \mathrm{~min}-$ utes), the GAPDH block is released and the metabolic flux is re-established [71,72]. The transcriptional response occurs in discrete temporal steps; in yeast, upregulation of the first mRNA targets is detected 3-5 minutes after stress induction. The subsequent transcriptional changes follow the route of the metabolic flux. First, enzymes metabolizing glucose-6-phosphate are upgregulated, enzymes catalyzing the more downstream reactions follow than step by step [73]. The details of how this process is timed remain obscure, but it is likely that metabolic and genetic feedback loops must cooperate closely during this dynamic transition.

\section{Concluding remarks}

In this review, we have highlighted the role of the metabolic network as a fundamental part of the cellular regulome. Its unique modular structure facilitates participation in both system-wide and pathway-specific regulatory processes. Global regulation is often mediated through enzymatic cofactors or general substrates which are responsible for most of the interconnectivity of the metabolic network, whereas specific responses are controlled by specialised intermediates.

Changes in the flux of a metabolic pathway can have multiple regulatory consequences. In yeast, over half of the transcriptome is altered when the cell undergoes metabolic oscillations [53]. Glycolysis remains the best understood example for a single pathway that contains multiple reporters. Transitions in glycolysis have an important regulatory function under oxidative stress, and target multiple pathways in cancer.

Currently, the Kyoto encyclopaedia of genes and genomes (http://www.genome.jp/kegg/) lists 16.056 metabolites and small molecules that take part in 8056 biochemical reactions, which are organised into 704 modules. Although most of our current knowledge derives from studies in $E$. coli and yeast, recent technological advancements have facilitated the discovery of the function and interconnectivity of metazoan networks. These experimental investigations have been extended through large-scale in silico reconstructions of metabolic networks that are largely based on sequencing data [9,74].

Elucidating metabolites with reporter function and their biochemical effectors, and the mechanism of their interactions with one another and other cellular networks (e.g. signalling cascades), is a challenging but necessary undertaking. These efforts will provide essential knowledge of cellular organisation and thus important targets for treatments of human disease.

\section{Competing interests}

We declare that no competing interests exist.

\section{Acknowledgments}

We regret that many important scientific findings could not be discussed in this review. We thank our colleagues for interesting discussions and the Max Planck Society for funding.

\section{References}

1 Caetano-Anolles, G. et al. (2009) The origin and evolution of modern metabolism. Int. J. Biochem. Cell Biol. 41, 285-297

2 Wagner, A. (2005) Robustness and Evolvability in Living Systems, Princeton University Press

3 Beckwith, J.R. and Zipser, D. (1970) The Lactose Operon, Cold Spring Harbor Laboratory Press

4 Patil, K.R. and Nielsen, J. (2005) Uncovering transcriptional regulation of metabolism by using metabolic network topology. Proc. Natl. Acad. Sci. U. S. A. 102, 2685-2689

5 Cakir, T. et al. (2006) Integration of metabolome data with metabolic networks reveals reporter reactions. Mol. Syst. Biol. 2, 50

6 Ravasz, E. et al. (2002) Hierarchical organization of modularity in metabolic networks. Science 297, 1551-1555

7 Shen-Orr, S.S. et al. (2002) Network motifs in the transcriptional regulation network of Escherichia coli. Nat. Genet. 31, 64-68

8 Papin, J.A. et al. (2004) Hierarchical thinking in network biology: the unbiased modularization of biochemical networks. Trends Biochem. Sci. 29, 641-647

9 Palsson, B. (2009) Metabolic systems biology. FEBS Lett 583 (24), 3900-3904 PMID: 19769971

10 Kreimer, A. et al. (2008) The evolution of modularity in bacterial metabolic networks. Proc. Natl. Acad. Sci. U. S. A. 105, 6976-6981

11 De La Fuente, I.M. et al. (2008) Global self-organization of the cellular metabolic structure. PLoS One 3, e3100

$12 \mathrm{Ma}$, W. et al. (2009) Defining network topologies that can achieve biochemical adaptation. Cell 138, 760-773

13 Marx, C.J. et al. (2005) Flux analysis uncovers key role of functional redundancy in formaldehyde metabolism. PLoS Biol. 3, e16

14 Kashtan, N. et al. (2007) Varying environments can speed up evolution. Proc. Natl. Acad. Sci. U. S. A. 104, 13711-13716

15 Parter, M. et al. (2007) Environmental variability and modularity of bacterial metabolic networks. BMC Evol. Biol. 7, 169

16 Blank, L.M. et al. (2005) Large-scale 13C-flux analysis reveals mechanistic principles of metabolic network robustness to null mutations in yeast. Genome Biol. 6, R49

17 Ishii, N. et al. (2007) Multiple high-throughput analyses monitor the response of E. coli to perturbations. Science 316, 593-597

18 Kuepfer, L. et al. (2005) Metabolic functions of duplicate genes in Saccharomyces cerevisiae. Genome Res. 15, 1421-1430

19 Hillenmeyer, M.E. et al. (2008) The chemical genomic portrait of yeast: uncovering a phenotype for all genes. Science 320, 362-365

20 Daran-Lapujade, P.et al. (2007) The fluxes through glycolytic enzymes in Saccharomyces cerevisiae are predominantly regulated at posttranscriptional levels. Proc. Natl. Acad. Sci. U. S. A. 104, 1575315758

21 Brauer, M.J. et al. (2005) Homeostatic adjustment and metabolic remodeling in glucose-limited yeast cultures. Mol. Biol. Cell 16, 2503-2517

22 Jensen, K.F. and Pedersen, S. (1990) Metabolic growth rate control in Escherichia coli may be a consequence of subsaturation of the macromolecular biosynthetic apparatus with substrates and catalytic components. Microbiol. Rev. 54 (2), 89-100 PMID: 1694554

23 Castrillo, J.I. et al. (2007) Growth control of the eukaryote cell: a systems biology study in yeast. J. Biol. 6, 4

24 Ralser, M. et al. (2009) Metabolic reconfiguration precedes transcriptional regulation in the antioxidant response. Nat. Biotechnol. 27, 604-605

25 Wullschleger, S. et al. (2006) TOR signaling in growth and metabolism. Cell 124, 471-484

26 Steinberg, G.R. and Kemp, B.E. (2009) AMPK in Health and Disease. Physiol. Rev. 89 (3), 1025-1078 PMID: 19584320

27 Heeren, G. et al. (2009) The mitochondrial ribosomal protein of the large subunit, Afo1p, determines cellular longevity through mitochondrial back-signaling via TOR1. Aging 1, 622-636 
28 Wilson, D.N. and Nierhaus, K.H. (2007) The weird and wonderful world of bacterial ribosome regulation. Crit. Rev. Biochem. Mol. Biol. 42, 187-219

29 Wellen, K.E. et al. (2009) ATP-citrate lyase links cellular metabolism to histone acetylation. Science 324, 1076-1080

30 Williams, R.B. et al. (2008) Epigenetic remodeling of the fungal secondary metabolome. Org. Biomol. Chem. 6, 1895-1897

31 Tanny, J.C. et al. (1999) An enzymatic activity in the yeast Sir2 protein that is essential for gene silencing. Cell 99, 735-745

32 Lin, S.J. et al. (2002) Calorie restriction extends Saccharomyces cerevisiae lifespan by increasing respiration. Nature 418, 344-348

33 Gavin, A.C. et al. (2002) Functional organization of the yeast proteome by systematic analysis of protein complexes. Nature 415, 141-147

34 Matecic, M. et al. (2002) SIR2-induced inviability is suppressed by histone H4 overexpression. Genetics 162, 973-976

35 Ralser, M. et al. (2009) Interfering with glycolysis causes Sir2dependent hyper-recombination of Saccharomyces cerevisiae plasmids. PLoS One 4, e5376

36 Fontana, L. (2009) The scientific basis of caloric restriction leading to longer life. Curr. Opin. Gastroenterol. 25, 144-150

37 Ying, W. (2008) NAD+/NADH and NADP+/NADPH in cellular functions and cell death: regulation and biological consequences. Antioxid. Redox. Signal. 10, 179-206

38 Pollak, N. et al. (2007) The power to reduce: pyridine nucleotides - small molecules with a multitude of functions. Biochem. J. 402, 205-218

39 Kun, E. et al. (2004) Regulation of the enzymatic catalysis of poly(ADPribose) polymerase by dsDNA, polyamines, $\mathrm{Mg} 2+, \mathrm{Ca} 2+$, histones $\mathrm{H} 1$ and H3, and ATP. Biochemistry 43 (1), 210-216 PMID: 14705947

40 Timinszky, G. et al. (2009) A macrodomain-containing histone rearranges chromatin upon sensing PARP1 activation. Nat. Struct Mol. Biol. 16, 923-929

41 Ahel, D. et al. (2009) Poly(ADP-ribose)-dependent regulation of DNA repair by the chromatin remodeling enzyme ALC1. Science $325,1240-1243$

42 Winkler, W.C. and Breaker, R.R. (2005) Regulation of bacterial gene expression by riboswitches. Annual review of microbiology 59, 487-517

43 Henkin, T.M. (2008) Riboswitch RNAs: using RNA to sense cellular metabolism. Genes Dev 22, 3383-3390

44 Miranda-Rios, J. (2007) The THI-box riboswitch, or how RNA binds thiamin pyrophosphate. Structure 15, 259-265

45 Theobald, U. et al. (1993) In vivo analysis of glucose-induced fast changes in yeast adenine nucleotide pool applying a rapid sampling technique. Anal Biochem. 214, 31-37

46 Pinson, B. et al. (2009) Metabolic intermediates selectively stimulate transcription factor interaction and modulate phosphate and purine pathways. Genes Dev. 23, 1399-1407

47 Kresnowati, M.T. et al. (2006) When transcriptome meets metabolome: fast cellular responses of yeast to sudden relief of glucose limitation. Mol. Syst. Biol. 2, 49

48 Guetsova, M.L. et al. (1997) The isolation and characterization of Saccharomyces cerevisiae mutants that constitutively express purine biosynthetic genes. Genetics 147, 383-397

49 Whitford, P.C. et al. (2009) Nonlocal helix formation is key to understanding S-adenosylmethionine-1 riboswitch function. Biophys. J. 96, L7-9

50 Loh, E. et al. (2009) A trans-Acting Riboswitch Controls Expression of the Virulence Regulator PrfA in Listeria monocytogenes. Cell 139, 770 779

51 Fujita, Y. (2009) Carbon catabolite control of the metabolic network in Bacillus subtilis. Biosci Biotechnol Biochem. 73 (2), 245-259 PMID: 19202299
52 Dahl, M.K. (2002) CcpA-independent carbon catabolite repression in Bacillus subtilis. J. Mol. Microbiol.Biotechnol. 4, 315-321

$53 \mathrm{Tu}$, B.P. et al. (2005) Logic of the yeast metabolic cycle: temporal compartmentalization of cellular processes. Science 310, 1152-1158

$54 \mathrm{Hsu}$, P.P. and Sabatini, D.M. (2008) Cancer cell metabolism: Warburg and beyond. Cell 134, 703-707

55 Bellance, N. et al. (2009) Mitochondria: from bioenergetics to the metabolic regulation of carcinogenesis. Front. Biosci. 14, 4015-4034

56 Schafer, Z.T. et al. (2009) Antioxidant and oncogene rescue of metabolic defects caused by loss of matrix attachment. Nature 461, 109-113

57 Mazurek, S. et al. (2005) Pyruvate kinase type M2 and its role in tumor growth and spreading. Semin. Cancer Biol. 15 (4), 300-308 PMID: 15908230

58 Christofk, H.R. et al. (2008) The M2 splice isoform of pyruvate kinase is important for cancer metabolism and tumour growth. Nature 452,230 233

59 Becker, J. et al. (2008) Metabolic responses to pyruvate kinase deletion in lysine producing Corynebacterium glutamicum. Microb. Cell Fact. 7, 8

60 Cunningham, D.S. et al. (2009) Pyruvate kinase-deficient Escherichia coli exhibits increased plasmid copy number and cyclic AMP levels. $J$. Bacteriol. 191, 3041-3049

61 Bakowski, D. and Parekh, A.B. (2007) Regulation of store-operated calcium channels by the intermediary metabolite pyruvic acid. Curr. Biol. 17, 1076-1081

62 Roderick, H.L. and Cook, S.J. (2008) Ca2+ signalling checkpoints in cancer: remodelling $\mathrm{Ca} 2+$ for cancer cell proliferation and survival. Nat. Rev. Cancer. 8 (5), 361-375 PMID: 18432251

63 Hashimoto, T. et al. (2007) Lactate sensitive transcription factor network in L6 cells: activation of MCT1 and mitochondrial biogenesis. Faseb. J. 21, 2602-2612

64 Yalcin, A. et al. (2009) Regulation of glucose metabolism by 6phosphofructo-2-kinase/fructose-2,6-bisphosphatases in cancer. Exp. Mol. Pathol. 86, 174-179

65 Lee, M.N. et al. (2009) Glycolytic flux signals to mTOR through glyceraldehyde-3-phosphate dehydrogenase-mediated regulation of Rheb. Mol. Cell Biol. 29, 3991-4001

66 Janero, D.R. et al. (1994) Hydroperoxide-induced oxidative stress impairs heart muscle cell carbohydrate metabolism. Am. J. Physiol. 266, C179-188

67 Grant, C.M. (2008) Metabolic reconfiguration is a regulated response to oxidative stress. J. Biol. 7, 1

68 Shenton, D. and Grant, C.M. (2003) Protein S-thiolation targets glycolysis and protein synthesis in response to oxidative stress in the yeast Saccharomyces cerevisiae. Biochem. J. 374, 513-519

69 Ralser, M. et al. (2007) Dynamic rerouting of the carbohydrate flux is key to counteracting oxidative stress. J. Biol. 6, 10

70 Godon, C. et al. (1998) The $\mathrm{H} 2 \mathrm{O} 2$ stimulon in Saccharomyces cerevisiae. J. Biol. Chem. 273, 22480-22489

71 Grant, C.M. et al. (1999) Differential protein S-thiolation of glyceraldehyde-3-phosphate dehydrogenase isoenzymes influences sensitivity to oxidative stress. Mol. Cell Biol. 19, 2650-2656

72 Colussi, C. et al. (2000) H2O2-induced block of glycolysis as an active ADP-ribosylation reaction protecting cells from apoptosis. FASEB $J$. $14,2266-2276$

73 Chechik, G. et al. (2008) Activity motifs reveal principles of timing in transcriptional control of the yeast metabolic network. Nat. Biotechnol. 26, 1251-1259

74 Price, N.D. et al. (2004) Genome-scale models of microbial cells: evaluating the consequences of constraints. Nat. Rev. Microbiol. 2, 886-897 